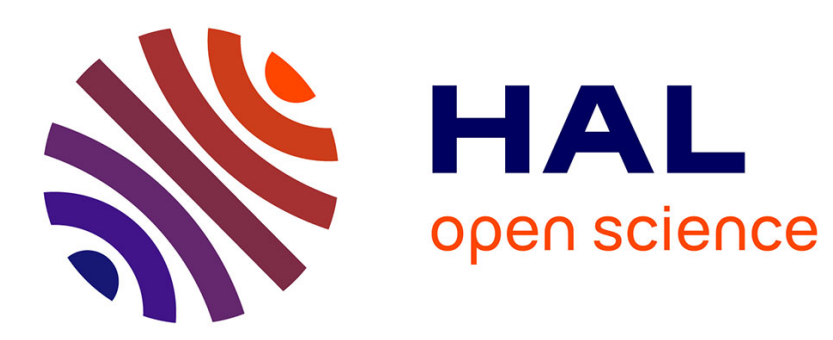

\title{
Particle Swarm Optimization Method in Thermoacoustic Problems
}

\author{
Hussein Chaitou, Philippe Nika, Guillaume Layes
}

\section{To cite this version:}

Hussein Chaitou, Philippe Nika, Guillaume Layes. Particle Swarm Optimization Method in Thermoacoustic Problems. ACOUSTICS Hong Kong, May 2012, Hong Kong, Hong Kong SAR China. hal-03223587

\section{HAL Id: hal-03223587 \\ https://hal.science/hal-03223587}

Submitted on 11 May 2021

HAL is a multi-disciplinary open access archive for the deposit and dissemination of scientific research documents, whether they are published or not. The documents may come from teaching and research institutions in France or abroad, or from public or private research centers.
L'archive ouverte pluridisciplinaire HAL, est destinée au dépôt et à la diffusion de documents scientifiques de niveau recherche, publiés ou non, émanant des établissements d'enseignement et de recherche français ou étrangers, des laboratoires publics ou privés. 


\title{
ACOUSTICS 2012 HONG KONG
}

\section{Particle Swarm Optimization Method in Thermoacoustic Problems}

\author{
Hussein Chaitoul, Philippe Nika and Guillaume Layes \\ Institut FEMTO-ST / UMR CNRS 6174 \\ Parc technologique, 2, avenue Jean Moulin, 90000 Belfort, France
}

\begin{abstract}
Thermoacoustic engine systems convert heat power into acoustic power which is useful to pump heat or to generate electricity. To construct a robust and useful thermoacoustic device, both the acoustic power produced and the exergetic efficiency of this device should have acceptable and meaningful values. In order to attain this objective, an optimization study is strongly recommended and required. In the literature of thermoacoustic research, we found only some limited synthetic optimization methods. This paper presents a new study that incorporates the Particle Swarm Optimization (PSO) method for the first time in the thermoacoustic research in order to optimize the two objective functions, i.e. the acoustic power and the exergetic efficiency. The importance of using the PSO method in thermoacoustic research is highlighted and extensively investigated. In addition, significant conclusions, which are useful for the design of new thermoacoustic engines, are discussed.
\end{abstract}

Keywords: Thermoacoustic, Engine, Particle Swarm Optimization.

\section{Introduction}

The thermoacoustic phenomenon was first explained qualitatively a long time ago, in the year 1878 , by Rayleigh": "if heat be given to the air at the moment of greatest condensation, i.e. greatest density, or be taken from it at the moment of greatest rarefaction, the vibration is encouraged". However, the first concrete theory and quantitatively accurate understanding to the thermoacoustic phenomenon was given a century later by Rott ${ }^{2}$. Based on Rott's thermoacoustic approximation, Swift from Los Alamos National Laboratory and Garret from PEN State University have successfully led the field by making the first devices that produce a useful acoustic work ${ }^{3,4}$. Hence, the interest to thermoacoustic systems has been arisen and expanded.

In fact, thermoacoustic systems have a lot of benefits. As an example, they can use any external energy sources, they have no or few moving parts, they are friendly environmental and they are low cost machines. Thus, to build a thermoacoustic device, it is sufficient to have a porous medium, stack or regenerator, sandwiched between hot and cold heat exchangers inside a resonator. Despite all these advantages, the exergetic efficiency of these devices is still relatively low and need to be improved by keeping, at the same time, an acceptable and meaningful value of the acoustic power produced by these devices. To reach this objective, the Particle Swarm Optimization (PSO) method is investigated in the present work. In contrast to the limited optimization methods recently appeared in the literature ${ }^{5-7}$, the PSO method performs very well in optimizing a complex problem with a large number of parameters, such as thermoacoustic problems, without needing a huge amount of calculation time and space in the actual computers. Also, the PSO method can do a multi-objective optimization, i.e. exergetic efficiency and acoustic power produced by a thermoacoustic device, for the same problem in one single simulation.

The reminder of this paper is organized as follows. Section 2, an introduction to the PSO method is presented. In section 3 , the thermoacoustic equations, developed by Rott and written by $\mathrm{Swift}^{8}$, are

\footnotetext{
${ }^{1}$ hussein.chaitou@univ-fcomte.fr
} 
rewritten in a dimensionless numbers. The PSO method is applied to optimize the exergetic efficiency, the acoustic power and their product in section 4 . This paper is ended up with conclusions and perspectives for future works.

\section{Introduction to the Particle Swarm Optimization method}

PSO is an iterative method that tries to maximize or minimize a function or problem in a known search-space of dimension $N$. Every problem has multiple candidate solutions, called particles, which are characterized by their positions and velocities over mathematical formulae. The PSO was invented by Kennedy and Eberhart ${ }^{9}$ in 1995, inspired by social behavior of bird flocking or fish schooling.

Suppose the function to be optimized, $f$, is defined as:

$$
f: \begin{cases}\mathbb{R}^{N} & \rightarrow \mathbb{R} \\ \vec{x} & \rightarrow f(\vec{x})\end{cases}
$$

Where $\vec{x}$ is the position vector of one particle. Then, let $P$ be the number of particles in the swarm, where each particle has a position vector of dimension $N$. So, the position vector of a particle $i$ is defined as $\vec{x}_{i}$, where $i=1, \ldots, P$, and $\vec{v}_{i}$ is its velocity.

Let $k$ be the number of iterations, then the algorithm for maximizing a function can be summarized as:

I- Initialize the position vector, $\vec{x}_{0}^{i}$, the function $f_{0}^{i}\left(\vec{x}_{0}^{i}\right)$ and the velocity vector, $\vec{v}_{0}^{i}$, for each particle

II- For each iteration, $j=1, \ldots, k$

○ For each particle, $i=1, \ldots, P$

○ Evaluate the fitness value of the function, $f_{j}^{i}\left(\vec{x}_{j}^{i}\right)$ at position $\vec{x}_{j}^{i}$,

○ If $f_{j}^{i}\left(\vec{x}_{j}^{i}\right)>f_{\text {best }}^{i}\left(\vec{x}_{\text {best }}^{i}\right)$, then $f_{\text {best }}^{i}\left(\vec{x}_{\text {best }}^{i}\right)=f_{j}^{i}\left(\vec{x}_{j}^{i}\right)$ and $\vec{x}_{\text {best }}^{i}=\vec{x}_{j}^{i}$, where $f_{\text {best }}^{i}\left(\vec{x}_{\text {best }}^{i}\right)$ is the best value retained by a particle $i$ at position $\vec{x}_{\text {best }}^{i}$

○ If $f_{j}^{i}\left(\vec{x}_{j}^{i}\right)>f_{\text {best }}^{g}\left(\vec{x}_{\text {best }}^{g}\right)$, then $f_{\text {best }}^{g}\left(\vec{x}_{\text {best }}^{g}\right)=f_{j}^{i}\left(\vec{x}_{j}^{i}\right)$ and $\vec{x}_{\text {best }}^{g}=\vec{x}_{j}^{i}$, where $f_{\text {best }}^{g}\left(\vec{x}_{\text {best }}^{g}\right)$ is the global best value retained at position $\vec{x}_{\text {best }}^{g}$

$\circ \quad$ Update particle velocity, $\vec{v}_{j+1}^{i}$, by using the appropriate mathematical formulae

○ Update particle position, $\vec{x}_{j+1}^{i}$, where $\vec{x}_{j+1}^{i}=\vec{x}_{j}^{i}+\vec{v}_{j+1}^{i}$

$\circ$ If stopping conditions are satisfied, go to step III

III- Report results and terminate.

In this paper, the constriction method is used to calculate the update particle's velocity ${ }^{10,11}$ :

$$
\vec{v}_{j+1}^{i}=0.729 \vec{v}_{j}^{i}+1.494 \vec{U}_{1}(0,1) \times\left(\vec{x}_{\text {best }}^{i}-\vec{x}_{j}^{i}\right)+1.494 \vec{U}_{2}(0,1) \times\left(\vec{x}_{\text {best }}^{g}-\vec{x}_{j}^{i}\right)
$$

Where, $\vec{U}_{1}(0,1)$ and $\vec{U}_{2}(0,1)$ are two random vectors in which each component goes from zero to one. The velocity $\vec{v}$ is limited to $\left[\vec{x}_{\min }, \vec{x}_{\text {max }}\right]$. This constriction method has a high success rate ${ }^{12}$, and hence, it decreases the risk of premature convergence to non-optimal points.

\section{Dimensionless number of thermoacoustic equations}

The thermoacoustic equations, derived by Rott and written by Swift ${ }^{8}$, are rewritten in dimensionless numbers by using Table 1 . Then, as we are only interested in what is happening inside the stack, the studying zone is reduced to the stack. Howerver, the position of the studying zone varies in the resonator. So the position in the stack, $x^{*}$, varies between $\left[x_{c}^{*}-\frac{L^{*}}{2} ; x_{c}^{*}+\frac{L^{*}}{2}\right]$, (see Figure 1), where $x_{c}^{*}$ is the center of 
the stack, and $L^{*}$ is the length of the stack. Besides, the oscillating pressure propagating, in an ideal gas in the direction $x^{*}$ of a channel with a cross-sectional area A, is supposed to be:

$$
p_{a}^{*}=D R\left((1-\tau) e^{j 2 \pi x^{*}}+\tau e^{-j 2 \pi x^{*}}\right)
$$

where, $\tau$ is the traveling-standing-wave ratio, $0 \leq \tau \leq 1$. It is equal to 1 for a pure traveling-wave, 0.5 for a pure standing-wave, and 0 for a pure reflecting traveling-wave, $D R$ is the drive ratio, $D R=\frac{p}{\bar{p}_{g}}, p$ is the pressure amplitude and $\bar{p}_{g}$ is the mean pressure.

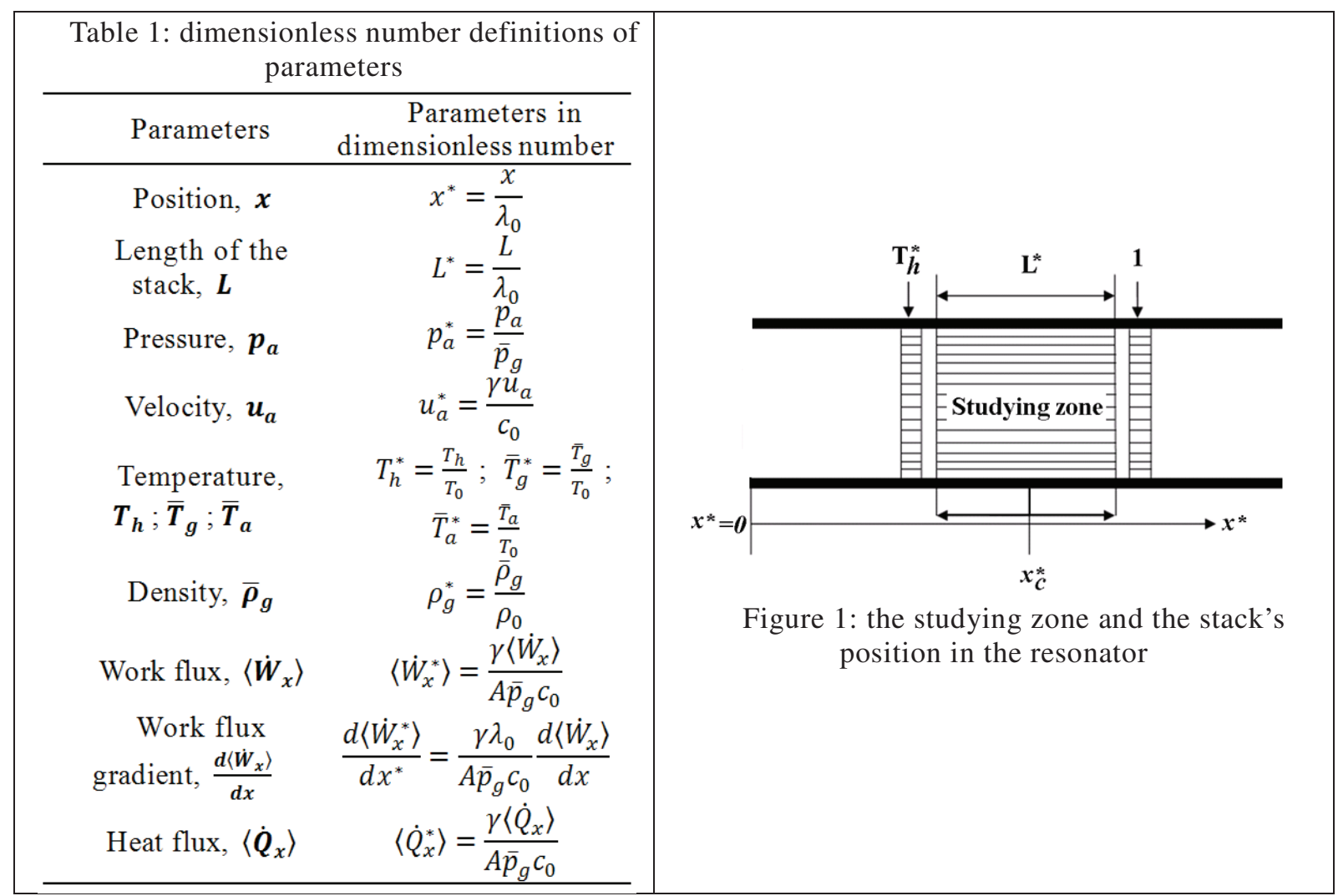

The derivation of the time-averaged acoustic power $d\left\langle\dot{W}_{x}^{*}\right\rangle$ produced in a length $d x^{*}$ of the channel is given as:

$$
\frac{d\left\langle\dot{W}_{x}^{*}\right\rangle}{d x^{*}}=\frac{1}{\bar{T}_{g}^{*}} \frac{d \bar{T}_{g}^{*}}{d x^{*}} R\left[G_{0}\right]\left\langle\dot{W}_{x}^{*}\right\rangle+\frac{1}{2 \bar{T}_{g}^{*}} \frac{d \bar{T}_{g}^{*}}{d x^{*}} I\left[-G_{0}\right] I\left[\tilde{p}_{a}^{*} \bar{u}_{a}^{*}\right]-\frac{\lambda_{0} \gamma \bar{p}_{g}}{2 c_{0} r_{t}}\left|p_{a}^{*}\right|^{2}-\frac{\lambda_{0} c_{0} r_{v}}{2 \gamma \bar{p}_{g}}\left|u_{a}^{*}\right|^{2}
$$

where, $p_{a}^{*}$ and $u_{a}^{*}$ are the oscillating pressure and velocity, $\bar{T}_{g}^{*}$ is the gas mean temperature which is supposed to be linear throughout the stack, $\bar{T}_{g}^{*}=\frac{\bar{T}_{g}}{T_{0}}=T_{h}^{*}+\frac{\left(x^{*}-x_{c}^{*}+\frac{L^{*}}{2}\right)}{L^{*}}\left(1-T_{h}^{*}\right)$, and its axial thermal gradient $\frac{d \bar{T}_{g}^{*}}{d x^{*}}=\frac{\left(1-T_{h}^{*}\right)}{L^{*}}, r_{v}$ and $\frac{1}{r_{t}}$ are the acoustic viscous resistance and the acoustic thermal resistance, respectively, $r_{v}=\omega \bar{\rho}_{g} \frac{\mathfrak{\Im}\left(-g_{0}\left(s_{*}\right)\right)}{\left|1-g_{0}\left(s_{*}\right)\right|^{2}}$ and $\frac{1}{r_{t}}=\frac{\gamma-1}{\gamma} \frac{\omega}{\bar{p}_{g}} \mathfrak{J}\left(-g_{0}\left(s_{*} \sqrt{P r}\right)\right), G_{0}$ is a combined of the spatial averaged thermoacoustic functions, $G_{0}=\frac{\left(g_{0}\left(s_{*}\right)-g_{0}\left(s_{*} \sqrt{P r}\right)\right)}{(\operatorname{Pr}-1)\left(1-g_{0}\left(s_{*}\right)\right)}, g_{0}\left(s_{*}\right)$ is the spatial averaged of a complex 
thermoacoustic function which depends on the specific channel geometric, for two infinite parallel plates, $g_{0}\left(s_{*}\right)=\frac{\tanh \left(s_{*}\right)}{s_{*}}, s_{*}=\frac{r_{h}}{\delta_{v}} \sqrt{2 j}, \quad \delta_{v}$ is the viscous penetration depth, $\operatorname{Pr}$ is the Prandtl number, $\bar{\rho}_{g}$ is the mean density for an ideal gas, $\omega$ is the angular frequency, $\gamma$ is isobaric to isochoric specific heat ratio, $c_{0}$ is the sound speed at $T_{0}, r_{h}$ is the hydraulic radius of the stack, $R[]$ and $I[]$ are the real and imaginary parts, the tilde represents the conjugate number.

The heat flux, $\left\langle\dot{Q}_{x}^{*}\right\rangle$, which is a combination of two terms, an internal heat flux and a thermal-conduction, is equal to:

$$
\left\langle\dot{Q}_{x}^{*}\right\rangle=\left(\frac{c_{0}}{2 \omega \lambda_{0}} \frac{1}{\rho_{g}^{*}} \Im\left[\frac{d \tilde{p}_{a}^{*}}{d x^{*}} p_{a}^{*}\left(\tilde{g}_{0}\left(s_{*}\right)-\psi\right)\right]+\frac{\gamma \bar{p}_{g} T_{0}}{c_{0} \lambda_{0}^{3}} k_{e q}^{*} \frac{1}{\rho_{g}^{*}} \frac{d \bar{T}_{g}^{*}}{d x^{*}}\right)-\frac{\gamma T_{0}}{\bar{p}_{g} c_{0} \lambda_{0}}\left[(1-\varepsilon) k_{s} \frac{d \bar{T}_{s}^{*}}{d x^{*}}+\varepsilon k_{g} \frac{d \bar{T}_{g}^{*}}{d x^{*}}\right]
$$

where, $k_{e q}^{*}=\frac{c_{p g} \Im[\psi]}{2 \omega^{3} \rho_{0}(1-\operatorname{Pr})}\left|\frac{d p_{a}^{*}}{d x^{*}}\right|^{2}, \rho_{g}^{*}=\frac{\bar{\rho}_{g}}{\rho_{0}}=\frac{T_{0}}{\overline{\bar{T}}_{g}}=\frac{1}{\overline{\bar{T}}_{g}^{*}}$ and $\rho_{0}=\frac{\bar{p}_{g}}{r T_{0}}, \quad \psi=\tilde{g}_{0}\left(S_{*}\right)+\frac{\left(g_{0}\left(s_{*} \sqrt{P r}\right)-\tilde{g}_{0}\left(s_{*}\right)\right)}{(1+\operatorname{Pr})}$ and $c_{p g}$ is the isobaric heat capacity.

Approximately, the acoustic power produced or consumed by a stack or a regenerator can be calculated as the sum, throughout the stack, of the derivation of the time-averaged acoustic power $d\left\langle\dot{W}_{x}^{*}\right\rangle$ produced in a length $d x^{*}$, so:

$$
\Delta\left\langle\dot{W}^{*}\right\rangle=\left.\frac{L^{*}}{N} \sum_{i=1}^{N}\left(\frac{1}{\bar{T}_{g}^{*}} \frac{d \bar{T}_{g}^{*}}{d x^{*}} R\left[G_{0}\right]\left\langle\dot{W}_{x}^{*}\right\rangle+\frac{1}{2 \bar{T}_{g}^{*}} \frac{d \bar{T}_{g}^{*}}{d x^{*}} I\left[-G_{0}\right] I\left[\tilde{p}_{a}^{*} \bar{u}_{a}^{*}\right]-\frac{\lambda_{0} \gamma \bar{p}_{g}}{2 c_{0} r_{t}}\left|p_{a}^{*}\right|^{2}-\frac{\lambda_{0} c_{0} r_{v}}{2 \gamma \bar{p}_{g}}\left|u_{a}^{*}\right|^{2}\right)\right|_{c i} ^{*}
$$

Where, $N$ is the discretization number of the stack, $x_{c i}^{*}$ is the center position of the $\mathrm{i}^{\text {th }}$ interval step of the stack.

Supposing that a system receives a quantity $\dot{Q}_{h}^{*}$ of heat from a heat source which has an imposed hot temperature $T_{h}^{*}$, and the system produces a quantity $-\Delta\left\langle\dot{W}^{*}\right\rangle$ of acoustic power, then the exergetic efficiency is defined as:

$$
\eta_{e x}=\frac{-T_{h}^{*} \Delta\left\langle\dot{W}^{*}\right\rangle}{\left(T_{h}^{*}-1\right) \dot{Q}_{h}^{*}}
$$

Where, $\left\langle\dot{Q}_{h}^{*}\right\rangle$ is the heat added to the system at position $x_{h}^{*}$ and can be calculated by using Eq. (5), and $\Delta\left\langle\dot{W}^{*}\right\rangle$ is determined by using Eq. (6).

\section{Optimization using PSO method}

We are interesting in maximizing three functions. The first one is the exergetic efficiency of the thermoacoustic engine, $\eta_{e x}$, the second one is the acoustic power produced by the stack of a thermoacoustic engine, $\Delta\left\langle\dot{W}^{*}\right\rangle$. The last function is the product between the acoustic power produced by the stack and the exergetic efficiency, $\eta_{\mathrm{ex}} \times \Delta\left\langle\dot{\mathrm{W}}^{*}\right\rangle$ that is useful specially to find a tradeoff between the best of $\eta_{\text {ex }}$ and $\Delta\left\langle\dot{W}^{*}\right\rangle$, because, as we will see later, when $\eta_{\text {ex }}$ is in its maximum, $\Delta\left\langle\dot{W}^{*}\right\rangle$ has a low value, and vice versa, so, it is better to design a thermoacoustic device based on this function.

In this paper, we concern ourselves by studying a 3D search-space dimension problem composed from, the hydraulic radius of a parallel-plate stack, $r_{h}$, the center of the stack in the resonator, $x_{c}^{*}$ and the traveling-standing-wave ratio, $\tau$. Where, $x_{c}^{*} \in\left[\frac{L^{*}}{2} ; \frac{L^{*}}{2}+0.5\right], r_{h} \in[0.15 ; 0.31] \mathrm{mm}$ and $\tau \in[0.5 ; 1]$. These three parameters are studied to see their influence on the maximum cost functions mentioned above, $\eta_{\mathrm{ex}}$, $\Delta\left\langle\dot{\mathrm{W}}^{*}\right\rangle$ and $\eta_{\mathrm{ex}} \times \Delta\left\langle\dot{\mathrm{W}}^{*}\right\rangle$. 
Table 2: PSO evolution for each function optimized and their results

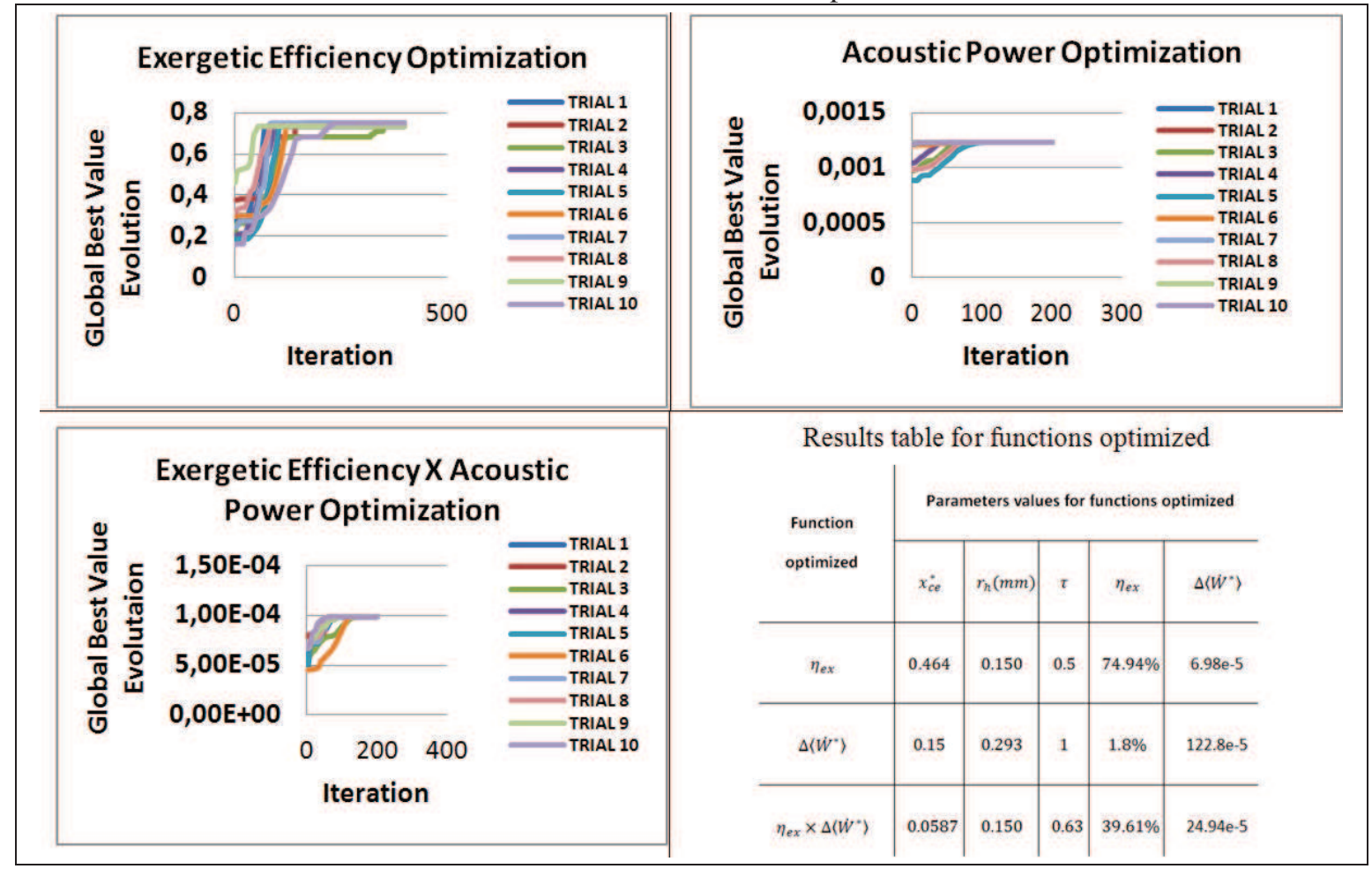

The other parameters are fixed as followed: the mean pressure is 25 bars, the hot and cold temperature are $893 \mathrm{~K}$ and $293 \mathrm{~K}$ respectively, the dimensionless length of the stack, $L^{*}=0.0025$, the drive ratio is chosen to $5 \%$ and the frequency is equal to $50 \mathrm{~Hz}$ while the gas used is Helium.

In fact, the drive ratio, hot temperature and cold temperature values determine the size of the hot and cold heat exchangers. In addition, the variation of $\tau$ means those boundary conditions are changing. So, once the optimization function is done, boundary conditions must be chosen to match the value of $\tau$.

The particle swarm optimization toolbox developed by Birge ${ }^{13}$ is used to optimize the desired functions. The number of particles is equal to 24. Then, each optimization function is run over 10 times.

As results show in Table 2, the best value of the exergetic efficiency is $74.94 \%$ whereas its acoustic power is relatively low at a value of $6.98 \mathrm{e}-5$, and its corresponding parameters are $\tau=0.5$, which means a resonator tube with two closed end boundary conditions, $r_{h}=0.15 \mathrm{~mm}<\delta_{t} \approx 0.3 \mathrm{~mm}$ and $x_{c}^{*} \approx 0.46$.

On the other hand, the best value of the acoustic power is $122.8 \mathrm{e}-5$ where the exergetic efficiency corresponding to this value is very low, $1.8 \%$ and while, $\tau=1$, which means a circled resonator tube, $r_{h} \approx \delta_{t} \approx 0.3 \mathrm{~mm}$ and $x_{c}^{*}=0.15$.

The optimization results of $\eta_{e x}$ and $\Delta\left\langle\dot{W}^{*}\right\rangle$ agreed with the work of Kang ${ }^{6}$. Obviously, the definition of $\tau$ in Kang work is different than the one in this paper. In fact, Kang found that the best value of the normalized acoustic power gain is when $\tau=0.68$, but this value is not matching the best value of the acoustic power itself. To calculate the acoustic power gain in the Kang's paper, we must multiply the normalized acoustic power gain by $\frac{p^{2}}{4 T \bar{\rho}_{g} c_{0}^{2}}\left(1+\tau^{2}\right)$. If this transfer condition is taken into account, we will find that the best value of the acoustic power gain is when the wave is a pure traveling, that is to say $\tau=1$ in this paper.

As we saw, the best value of exergetic efficiency leads to a relatively low acoustic power, while the best value of the acoustic power matches fairly low exergetic efficiency. So it might not be useful to design a thermoacoustic device based only on the best value of the exergetic efficiency or on the acoustic power. Hence, it is of prime importance to optimize the product between the exergetic efficiency and acoustic power as a tradeoff solution between the best values of the acoustic power and exergetic 
efficiency. Consecutively, the best value of this product is when $\tau=0.63, r_{h}=0.15 \mathrm{~mm}<\delta_{t} \approx 0.3 \mathrm{~mm}$ and $x_{c}^{*}=0.0587$ which corresponds to $\eta_{\mathrm{ex}}=39,61 \%$ and $\Delta\left\langle\dot{\mathrm{W}}^{*}\right\rangle=24.94 e-5$. In fact, this optimization function could give an explanation to the empirical study presented by Backhaus and Swift ${ }^{14}$.

\section{Conclusions and perspectives}

The PSO method has been successfully applied to the optimization of the acoustic power, the exergetic efficiency and their product of a thermoacoustic engine. In this first application PSO method to thermoacoustic problems, a simple model of a thermoacoustic engine was presented and studied. The acoustic pressure is supposed to be known, and only the variations of three parameters are studied, the stack's hydraulic radius, the stack's position in the resonator and the traveling-standing-wave ratio while other parameters are fixed.

As a result, the optimal solution of the acoustic power is obtained when the wave is a pure traveling and when the hydraulic radius is in the order of the thermal penetration depth. However, in this case, the exergetic efficiency is quite low. To reach highest exergetic efficiency, the thermoacoustic engine must work with a pure standing-wave, while the hydraulic radius has to be lower than the penetration depth and the corresponding acoustic power has to be low. As it is not much useful to build a thermoacoustic engine only based on higher exergetic efficiency with lower acoustic power or on higher acoustic power with lower exergetic efficiency, the product between acoustic power and exergetic efficiency is also studied. Such function is more realistic as it leads to both a good exergetic efficiency and acoustic power. The corresponding traveling-standing-wave ratio is in the order of 0.6 while the hydraulic radius is shorter than the thermal penetration depth.

Regarding the interesting results given by the use of the PSO method, future work will focus on studying more complicated thermoacoustic devices by trying to calculate the acoustic pressure instead of imposing it and to take into account a large search-space dimension problem.

\section{References and links}

1. Rayleigh L. The explanation of certain acoustical phenomena. Nature. 1878;18:319-321.

2. Rott N. Thermoacoustics. Advances in Applied Mechanics. 1980;20:135-175.

3. Swift GW. Thermoacoustic engines and refrigerators. Physics Today. 1995;48(7):22-28.

4. Garrett SL. Resource letter: Ta-1: thermoacoustic engines and refrigerators. American Journal of Physics. 2004;72(1):11-17.

5. Babaei H, Siddiqui K. Design and optimization of thermoacoustic devices. Energy Conversion and Management. 2008;49:3585-3598.

6. Kang H, Li Q, Zhou G. optimizing hydraulic radius and acoustic field of the thermoacoustic engine. Cryogenics. 2009;49(3-4):112-119.

7. Hu ZJ, Li ZY, Li Q, Li Q. Evaluation of thermal efficiency and energy conversion of thermoacoustic Stirling engines. Energy Conversion and Management. 2010;51(4):802-812.

8. Swift G. Thermoacoustics: A unifying perspective for some engines and refrigerators. 5 th ed.

(LANL, ed.).; 2001.

9. Kennedy J, Eberhart R. Particle Swarm Optimization. IEEE. 1995;4:1942-1948.

10. Clerc M. The swarm and the queen: towards a deterministic and adaptive particle swarm optimization. Proc. ICEC, Washington, DC. 1999:1951-1957.

11. Eberhart R, Shi Y. Particle Swarm Optimizatio: Developments Applications and Resources. IEEE. 2001:81-86.

12. Trelea IC. The particle swarm optimization algorithm: convergence analysis and parameter selection. Information Processing Letters. 2003;85:317-325.

13. Birge B. Particle Swarm Optimization Toolbox. MathWorks, optimization toolbox. 2006. Available at: http://www.mathworks.com/matlabcentral/fileexchange/7506 [Accessed March 11, 2011].

14. Backhaus S, Swift GW. A thermoacoustic-Stirling heat engine: Detailed study. The Journal of the Acoustical Society of America. 2000;107(6):3148-3166. 